\section{Focusing siRNA on the spleen}

\section{By Tracey Baas, Senior Editor}

Separate academic teams have collaborated with Alnylam Pharmaceuticals Inc. to target small interfering RNAs to immune cells residing in the spleen. The therapeutics silence inflammatory or antigen-presenting immune cells before they migrate to tissues where they can cause immunopathology. ${ }^{1,2}$

The first team, led by researchers from Harvard Medical School and the Massachusetts Institute of Technology, focused on using siRNA to inhibit the migration of inflammatory monocytes or progeny macrophages from the spleen to peripheral tissues. Those immune cells play a role in diseases such as atherosclerosis and cancer.

Because inflammatory monocyte and macrophage recruitment relies in part on CC chemokine receptor 2 (CCR2; CD192), the researchers hypothesized that knocking down the receptor would selectively inhibit migration of inflammatory cells without disrupting the noninflammatory subset of monocytes and macrophages.

In healthy mice, systemically delivered nanoparticles loaded with anti-CCR2 siRNA accumulated in the red pulp of the spleen. siRNA uptake was the highest in inflammatory monocytes, although the siRNAs also were taken up by neutrophils, noninflammatory monocytes, dendritic cells and macrophages.

In a mouse model of myocardial infarction, these nanoparticles decreased infarct size and the number of proinflammatory monocytes in the heart compared with small molecule inhibitors of CCR2.

Matthias Nahrendorf, a corresponding author on the paper, explained why siRNA might have this increased efficacy over small molecules: "Nanoparticle delivery can enrich the anti-CCR2 siRNA at the site of action, whereas the small molecule inhibitors are distributed throughout the body, which may lead to insufficient concentration at the receptor. In addition, small molecules can sometimes be less specific and block other receptors, whereas the siRNA approach targets one specific gene."

Nahrendorf is an assistant professor of radiology at the Massachusetts General Hospital Center for Systems Biology and Harvard Medical School.

In a mouse model of atherosclerosis, the nanoparticles reduced atherosclerotic lesion size and the number of proinflammatory monocytes in the lesions compared with nanoparticles loaded with control siRNA.

Compared with control nanoparticles, the anti-CCR2 nanoparticles decreased tumor growth and the number of tumor-associated macrophages in mice with lymphoma.

Finally, the nanoparticles extended the survival of pancreatic islet grafts in diabetic mice, whereas animals treated with control nanoparticles showed graft rejection.

Results were published in Nature Biotechnology.

"I like to think of our approach as a new class of cell-specific anti-inflammatory therapy," said Nahrendorf. "Since knockdown specifically affects inflammatory monocytes, our strategy should leave the functions of other immune cells intact to maintain immune homeostasis and defenses."

\section{Bigger is better}

Similar to the Massachusetts team, a group at The University of British Columbia also wanted to focus on knocking down targets in immune cells.

Their first step was finding a lipid formulation able to deliver siRNAs to immune cells in the spleen. Several lipids were tested. Within the subset, the team identified the lipid 1,2-dilinoleyl-4-(2dimethylaminoethyl)-[1,3]-dioxolane (DLinKC2-DMA), which previously had been used for hepatic delivery, ${ }^{3}$ to be the best.

The team reasoned that enlarging the particles would allow for better delivery to antigen-presenting cells (APCs) in the spleen, as they specialize in uptake of particles of various sizes, whereas the liver only allows passage of carriers $<100-150 \mathrm{~nm} .^{4}$

The researchers produced three sizes of nanoparticles, loaded with siRNA targeting either a liver-specific gene or a pan-leukocyte marker found in APCs in the spleen, and found that systemic administration of the largest particles to mice preferentially delivered siRNAs to the phagocytic APCs in the spleen. Silencing of the leukocyte marker was identical for all three sizes, whereas silencing of the liver gene decreased with increasing nanoparticle size.

Results were published in Molecular Therapy. The team also included researchers from AlCana Technologies Inc. Under a 2009 collaboration, Alnylam, AlCana and UBC are developing cationic lipid nanoparticles for the systemic delivery of RNAi therapeutics.

"The strategy of increasing the particle size demonstrated improved access to antigen-presenting cells as opposed to hepatocytes," said Tatiana Novobrantseva, associate director of research at Alnylam and an author on both studies. "There are still other modifications that can be employed, based on what we've learned from our work with hepatic gene silencing, such as adjusting 


\section{ANALYSIS}

lipid ratios and including cell-specific ligands into the nanoparticle formulation."

The company's siRNAs in Phase I testing target disease-related genes expressed in the liver. These include ALN-TTR01 to treat transthyretin (TTR)-mediated amyloidosis and ALN-PCS to treat hypercholesterolemia.

\section{Silence please}

For Alnylam, the ability to silence genes in immune cells could open up inflammatory and autoimmune indications.

John Rossi, chair of molecular and cellular biology at the Beckman Research Institute of City of Hope and cofounder of Dicerna Pharmaceuticals Inc., said the work in both manuscripts is "a significant advance, since targeting lymphocytes is always difficult. Future studies will need to evaluate both toxicity and efficacy in nonhuman primates. Multiple injections of the nanoparticle-siRNA combo are going to be required," which could lead to toxicity.

Niren Murthy, associate professor of biomedical engineering at both the Georgia Institute of Technology and the Emory University School of Medicine, agreed that the researchers are "going to have to look more closely at toxicity. Having said that, the work in mice where they silence Ccr2 in the spleen to impede monocyte migration and stop disease progression is a very intelligent idea. They avoid the bottleneck of trying to target immune cells in tissues that pose a challenge for siRNA delivery-cardiac tissue for myocardial infarction or aortic tissue for atherosclerosis."

A patent application has been filed for multiple indications and is available for licensing through Alnylam.

Baas, T. SciBX 4(41); doi:10.1038/scibx.2011.1134

Published online Oct. 20, 2011

\section{REFERENCES}

1. Leuschner, F. et al. Nat. Biotechnol.; published online Oct. 9, 2011; doi:10.1038/nbt.1989

Contact: Matthias Nahrendorf, Massachusetts General Hospital and Harvard Medical School, Boston, Mass. e-mail: mnahrendorf@mgh.harvard.edu

Contact: Ralph Weissleder, same affiliation as above e-mail: rweissleder@mgh.harvard.edu

2. Basha, G. et al. Mol. Ther; published online Oct. 4, 2011; doi:10.1038/mt.2011.190

Contact: Genc Basha, The University of British Columbia, Vancouver, British Columbia, Canada e-mail: gbasha@mail.ubc.ca

3. Semple, S.C. et al. Nat. Biotechnol. 28, 172-176 (2010)

4. Wisse, E. et al. Gene Ther. 15, 1193-1199 (2008)

\section{COMPANIES AND INSTITUTIONS MENTIONED}

AICana Technologies Inc., Vancouver, British Columbia, Canada Alnylam Pharmaceuticals Inc. (NASDAQ:ALNY), Cambridge, Mass. Beckman Research Institute at City of Hope, Duarte, Calif. Dicerna Pharmaceuticals Inc., Watertown, Mass.

Emory University School of Medicine, Atlanta, Ga. Georgia Institute of Technology, Atlanta, Ga. Harvard Medical School, Boston, Mass. Massachusetts General Hospital, Boston, Mass. Massachusetts Institute of Technology, Cambridge, Mass. The University of British Columbia, Vancouver, British Columbia, Canada 